Systematic Review of Interventions Designed to Teach Imitation to Young Children with Disabilities

Jennifer R. Ledford

Peabody College, Vanderbilt University

Acknowledgement: I gratefully thank Georgia Pace, Annie Riedlinger, Kate Chazin, Evyn Zelnio, Priscilla Shin, Maeve Hasselman, and Catherine Simpson for their attention to detail in coding for this review. 


\begin{abstract}
This systematic review was designed to characterize current intervention research for increasing imitation for young children with disabilities, who often demonstrate delayed imitative behavior. Embedded and massed trial interventions were identified, with embedded interventions occurring during classroom activities (classroom-based embedded trials, CBET) or play activities (playbased embedded trials, PBET) and massed trial interventions occurring with in situ models (livemodel massed trials, LMMT) or video models (video-model massed trials, VMMT). Across intervention types, positive outcomes were more likely to occur when dependent variables were primary variables (i.e., not outcomes secondary to another dependent variable) and when they were context-bound (i.e., collected during intervention sessions). When only primary variables from high quality studies were considered, embedded trials (PBET, CBET) more often resulted in functional relations; however, this may be due to the fact that children in these studies had less pronounced imitation delays.
\end{abstract}




\section{Systematic Review of Interventions Designed to Teach Imitation to Young Children with Disabilities}

Imitation typically develops early in infancy and the frequency with which children use imitation during dyadic interactions increases throughout the first few years of life (Masur, 2006). Infants engage in increasingly complex imitative behaviors with caregivers and then toddlers begin to use dyadic imitation skills with their peers during play (Eckerman et al., 1989). Researchers have suggested that the increasingly complex use of imitation is both a product of and a process for cognitive development. That is, imitation is impacted by cognitive ability and increasing use of imitation facilitates cognitive growth (Masur, 2006). Imitation predicts a number of important outcomes for young children, including vocabulary use, play complexity, joint attention, and social reciprocity (McDuffie et al., 2005, 2007; Masur \& Eichorst, 2002; Rogers et al., 2003; Stone et al., 1997).

Young children with disabilities often display difficulties in adaptively using imitation in typical learning and play contexts (O’Neill \& Zeedyk, 2006; Rogers \& Williams, 2006; Whiten \& Brown, 1998). Some children have imitation delays that are associated with cognition (e.g., children with intellectual disabilities or developmental delays often display difficulties with imitation). However, cognitive status does not entirely account for imitation difficulties for children with autism (Rogers et al., 2003); this may be partially explained by the social impact of autism, which can include reduced attention to others and low social motivation (Vivanti \& Hamilton, 2014). This social explanation of imitation delays may also account for overgeneralized imitation for some children with autism (i.e., echolalia; Vivanti \& Hamilton, 2014), suggesting specific difficulties with identifying discriminative stimuli. That is, children can imitate but do not know when to do so. Regardless of the nature of the difficulty within and across disability types, improving imitation for young children is critical because it is a pivotal or keystone skill. That is, adaptive use of imitation leads to additional learning opportunities and the potential to acquire other important behaviors (e.g., joint attention, Stern; 1985). Thus, effectively and efficiently improving the use of imitation skills for young children with disabilities is critical (Ledford, Lane, \& Barton, 2019; Ledford \& Wolery, 2011).

Improving young children's imitation requires defining the term and identifying the situations in which we would like to improve these behaviors. Behavioral texts (i.e., Cooper et al., 2020) often employ a highly restrictive definition for imitative behavior, including only behavior that is topographically identical to the model, controlled by the physical characteristics of the model, occuring within a few seconds after the model, and novel to the imitator (e.g., a behavior never before emitted). Colloquially, imitation often refers to behaviors that only meet the first two criteria. Imitative behaviors are particularly difficult to assess because we may not know whether the model controls the behavior or whether other controlling variables are at play (e.g., Ledford \& Wolery, 2011; Masur, 2006). Moreover, curricula often identify that specific behaviors (i.e., particular topographies, like waving or tapping an object) should be identified and taught repeatedly until mastered. By design, these behaviors are not novel after the first teaching trial but are still typically referred to as being imitative.

In addition to complexities related to measurement, there are also complicating factors in that typical use of imitation may be associated with multiple functions. That is, there are numerous situations in which imitation might be lead to increased access to reinforcement for young children. For example, imitation of others may lead to social praise or tangible rewards for complying with a model prompt (e.g., "Do this"). It may also promote increased cultural and social correspondence and help children to recruit and maintain social attention (Grusec \& 
Abramovitz, 1982; Uzgiris, 1981). Compliance with a model prompt has been identified as important for efficient learning without use of physical prompts, which are restrictive and intrusive (Ledford et al., 2019) while imitating during play has been shown to increase positive peer responses (Grusec \& Abramovitch, 1982). Moreover, imitation can lead to opportunities to learn complex behaviors emitted by peers in inclusive settings (Rogers \& Williams, 2006); these opportunities are often used as a rationale for inclusive educational opportunities (Ledford \& Wolery, 2011). Given these important functions, it is unsurprising that many well-established curricula for young learners with disabilities include imitation of others as an early learning target (e.g., Early Start Denver Model; Rogers et al., 2010; Learning Experiences and Alternative Program for Preschoolers and their Parents [LEAP], Strain \& Bovey, 2011; Promoting the Emergence of Advanced Knowledge Relational Training System [PEAK], Dixon et al., 2014; Verbal Behavior Milestones Assessment and Placement Program [VB-MAPP], Sundberg, 2008).

Ledford and Wolery (2011) summarized the peer-reviewed literature assessing imitation interventions for children through aged 12 that was available through 2010. They identified four intervention types: discrete trial training (DTT) with peer and adult implementers, play-based interventions, and classroom-based interventions. DTT generally refers to the systematic implementation of antecedents (cues, prompting) and consequences (reinforcement) for a specified discrete behavior; it is often used synonymously with massed trials, which refers to systematic instruction that occurs with short inter-trial intervals (e.g., 10 trials occurring sequentially, with only a few seconds occurring between trials; Ledford et al., 2019). We will use the term massed trials to describe trials occurring in quick succession in a single session and will contrast that with the term embedded trials to mean trials that are interspersed during ongoing activities (such as those that occur during classroom or play activities).

The purpose of this systematic review is to update the Ledford \& Wolery review, addressing some of the limitations of that research, including: (a) conducting outcomes analysis only for studies with appropriate methodological rigor (i.e., three demonstrations of effect; Ledford \& Gast, 2018; What Works Clearinghouse, 2020), and (b) including both published and unpublished sources to reduce the likelihood of publication bias (Shadish, Zelinsky, Vevea, \& Kratochwill, 2016; Tincani \& Travers, 2018). Research questions guiding the review were: (a) What are the characteristics of participants, contexts and implementers, independent variables, and dependent variables included in studies designed to improve imitation for young children with disabilities? (b) To what extent do researchers identify functional relations between interventions and imitative behaviors? (c) In how many studies are maintenance and corollary variables measured, and to what extent are these outcomes positive? (d) How do characteristics and outcomes vary by study rigor, intervention type, and dependent variable type?

\section{Inclusion Criteria}

\section{Methods}

We included studies that (a) assessed the impact of an intervention designed to improve imitation, (b) included direct measurement of unprompted or spontaneous imitative behaviors unrelated to acquisition of a non-imitative behavior with multiple topographies taught simultaneously (i.e., did not include studies using model prompts to teach a specific discrete behavior; assessed imitation as a generalized operant), (c) included at least one participant aged 8 or younger identified as having a disability (single case studies) or having a sample with a mean of age 8 or younger, all of whom have a disability (group design studies), (d) inclusion of sufficient and interpretable data, defined as 3 data points per condition and graphed data for visual analysis (single case) or 10 participants per condition and pre-treatment equivalence 
(group design studies), and (e) inclusion of a baseline, control, or alternative intervention condition. A table listing these inclusion criteria according to the PICOS organization (O'Conner et al., 2008) is available via Supplemental Materials (Table S1).

\section{Search and Screening}

We conducted an initial search using PsycINFO and ProQuest Dissertations and Theses databases and the search string: ab(imitat* or peer model or observational learning) AND ab(teach or train or instruct) AND ab(disabilit* or autis* or retard* or developmental* delay*). We also conducted forward (via Google Scholar) and backward searches (from the reference list) for the Ledford and Wolery (2011) review. Finally, we conducted searches for each individual who authored more than one included study (as an author or dissertation committee member) using Google Scholar. For each search method, we first conducted title and abstract screening then conducted full text screenings for potentially eligible studies. A PRISMA diagram (Moher et al., 2009) depicting the total number of articles screened and included is available via Supplemental Materials (Figure S1). Group designs were eligible (as described above) but none were identified which met inclusion criteria.

\section{Descriptive Coding} Participants

We coded participant demographic information, including age, gender, race, ethnicity, and disability category. We also coded pre-intervention imitation assessment scores, when provided (most commonly via the Motor Imitation Scale, Stone et al., 1997), and reported inclusion criteria. When both MIS scales were reported (motor, object), we calculated a mean. When raw scores were reported, we converted them into percentages. We recorded peer- and adult-model MIS scores separately.

Because report of secondary diagnoses and deficits were rarely reported, we predicted whether significant language and cognitive delays were likely for each participant. We coded likely presence of a significant language impairment if there was evidence of receipt of speech language services, use of augmentative or alternative communication, use of only or primarily single words (if aged 2 or older), standard scores from language assessments of lower than 70, or age equivalent scores from language assessments more than 12 months lower than a participant's chronological age. We coded likely presence of a significant cognitive delay if authors included report of IQ scores of less than 70, diagnosis of intellectual disability, or report of severe or significant cognitive delay. We also coded whether authors reported that each participant engaged in challenging behavior or stereotypy.

\section{Intervention Type, Context, and Implementer}

We categorized interventions into five groups initially, and collapsed two categories for a total of four for the purposes of analyzing data. We defined play-based embedded trials (PBET) interventions as those including trials embedded in the context of play activities, with children imitating the implementer and often including components such as adult responsivity to child communication and adult imitation of child behaviors. We defined classroom-based embedded trials (CBET) interventions as those occurring during classroom activities, with children imitating non-implementing peers or siblings (e.g., teacher implemented intervention, peers served as models). We defined live-model massed trials (LMMT) interventions as implementerdirected massed trials occurring with peer or adult models; given only one study with peer models occurred, we collapsed these categories. We defined video-based massed trials (VMMT) interventions as implementer-directed massed trials presented via video recordings. Interventions could have been coded as an "other" type, but this did not occur. 
In addition to intervention type, we coded context of the intervention, including setting (home, school, clinic, research, not reported), instructional arrangement (individual, in a group with peers being taught imitation, in a group with peer models, not reported), and implementer (teaching staff, peer or sibling, parent, researcher, not reported).

\section{Dependent Variables and Measurement}

We identified each imitation dependent variable assessed in the context of a single case design and coded the topography type (verbal, object, gesture, multiple types), the number of actions taught, the measurement system (trial-based counts or percentages, free operant counts or rates, interval-recording, other). Verbal imitation was defined as any repeating any vocal sounds (e.g., saying "blue" after an adult models "blue), object imitation was defined as repeating any motor behavior that included object manipulation (e.g., rolling a car), and gesture imitation was defined as repeating any motor behavior without object manipulation (e.g., waving, clapping).

\section{Functional Relation Determination}

For included sources, we identified each design (e.g., A-B, multiple baseline across participants) and whether the design represented a comparison between two interventions or between one intervention and one non-intervention condition. Finally, we coded whether a functional relation could be assessed (sufficient number of potential demonstrations of effect and data points, lack of baseline changes like improving trends that preclude drawing conclusions). For designs for which the presence of a functional relation could be determined, we subsequently coded rigor and outcomes (described below). Due to the nature of data patterns (e.g., nonreversible behavior expected to change from zero or low levels to a criterion level), no effect size estimates with acceptable characteristics are appropriate (e.g., Ledford, Chazin, Gagnon, et al., 2020; Ledford \& Gast, 2018); thus, we did not conduct effect size calculations or meta-analysis.

\section{Rigor and Outcomes Coding}

We coded studies for which functional relation identification was possible using the Single Case Analysis and Review Framework (version 2.0; Ledford, Chazin, Lane, et al., 2020) to assess Overall Quality and Rigor $(Q \& R)$ and Outcomes. $Q \& R$ and Outcomes scores are graphed via a scatterplot, with high $Q \& R$ and Outcomes scores (top right quadrant) indicating believable positive effects. The SCARF evaluates rigor in the domains of dependent and independent variable reliability and data sufficiency. The SCARF evaluates quality in the domains of dependent variable, condition, and participant descriptions as well as ecological and social validity. SCARF $Q \& R$ scores are calculated by averaging rigor scores and quality scores and double-weighting rigor; the range of possible scores is $0-4$. We identified scores above 2.0 indicating high quality studies and scores of 1.8 as having adequate quality for interpreting outcomes; these values are consistent with previous research (Chazin et al., 2020; Zimmerman et al., 2018). Outcomes were evaluated via the SCARF based on visual analysis, with a score of 0 indicating no positive effects or detrimental behavior changes, scores of 1-2 indicating inconsistent effects, and scores of 3-4 indicating consistent positive effects. Scores of 3 and 4 are consistent with functional relation identification (Ledford, Chazin, Lane, et al., 2020).

The SCARF also allows for evaluation of the measurement of maintenance and generalization and related outcomes. Because imitation is a generalized behavior and all imitation outcomes measured in the context of single case designs, including those considered as generalization measures, were evaluated, we assessed non-imitation behaviors (e.g., social interactions) emitted by the children receiving imitation intervention, and refer to these as corollary variables. We otherwise followed SCARF guidelines for assessing maintenance and generalization (corollary variables) and graphed these variables via scatterplot. 
Because of a priori hypotheses regarding relations between certain measurement characteristics and outcomes, we coded each design eligible for SCARF coding as including generalized, partially-context bound, or wholly context-bound behaviors and as being primary or secondary variables. Generalized behaviors were defined as being measured in contexts that differed in terms of interaction type, materials, implementer, and setting (e.g., CBET instruction occurs in art activities and measurement of a generalized imitation variable occurs in free play; Yoder, Lloyd, \& Symons, 2018). Partially context-bound behaviors differed in terms of one or more but not all of the aforementioned variables - for example, measurement of imitation with different materials than those used in intervention sessions. Context-bound behaviors were those directly targeted and measured in the intervention context. Primary variables were identified using these criterion (in the listed order): (a) identified by authors as the primary variable or primary purpose of the study, (b) was the only variable, (c) was the only variable not identified as a generalization variable, or (d) was one of multiple variables but was presented as the first research question and initial graph. Secondary variables did not meet these criteria (e.g., primary purpose was parent training and imitative behavior of children was measured as a secondary outcome; primary variable was imitation during intervention and imitative behavior during free play was also measured).

\section{Interobserver Agreement}

All screening, descriptive coding, and SCARF coding was conducted by two independent researchers (e.g., 100\% double coded). The first author, an expert in early childhood special education and single case design and a BCBA-D developed screening and coding protocols, piloted and modified protocols, and conducted all primary screening and coding. One of seven special education graduate students conducted reliability screening or coding (with each graduate student assigned a specific domain such as screening, coding participant characteristics, SCARF). The secondary screener and secondary coders were trained via written and verbal instructions by the first author, practiced with non-included articles, received feedback, and then coded included articles in batches, with discrepancy discussions occurring between batches. Overall agreement was 99\% for screening (99-100\% across steps), 97\% (93-98\% across domains) for descriptive coding, and 93\% for rigor and outcomes coding (88-98\% across domains). See Table S2 in Supplemental Materials for more detailed agreement results.

\section{Participants}

\section{Results}

Across 39 sources (6 published duplicates of unpublished dissertations, 33 unique studies, see Sources included in Systematic Review in Supplemental Materials), 119 participants received imitation intervention. Gender was unreported for $9 \%$ of participants; when reported, $80 \%$ of participants were male and $20 \%$ were female. The average participant age was 3.4 years, with a range from 21 months to 7 years. Most $(n=79 ; 66 \%)$ participants were preschool aged (between 3.0-5.5 years), while $36(\mathrm{n}=30 \%)$ were toddlers (under 3.0 years) and 1 was schoolaged ( 7 years). Three participants were identified as being between 3 and 6 , but with no individual ages reported. Average age across intervention types was similar (3.2 VMMT; 3.3 PBET; 3.5 CBET; 3.7 LMMT). Little information was available regarding race and ethnicity (none reported for $71 \%$ of participants). When reported, 25 participants were White, 6 were Black, 5 were of Asian descent, 2 were multi-racial, and 2 were of Hispanic/Latino descent. Participants were commonly described as having autism or ASD (76\%), with other disability categories less commonly represented (intellectual disability $=13 \%$, developmental delay $=8 \%$, language impairment $=8 \%$ ). There were differences across intervention types, with only $21 \%$ of 
participants receiving CBET identified as having ASD and 50\% of participants identified as having developmental delays. Other intervention types included mostly participants with autism $(\mathrm{PBET}=90 \%, \mathrm{LMMT}=84 \%, \mathrm{VMMT}=100 \%)$.

Although language impairments were reported relatively infrequently as a specific disability, there was evidence of a language delay or deficit for nearly $80 \%$ of participants $(\mathrm{n}=$ 94; received speech/language services, use of AAC, used few words or only used single words, or had standard scores indicative of significant impairment), with $10 \%$ of participants having evidence of no impairments and language status of remaining participants being unclear (e.g., no evidence for or against impairment). Similarly, when evidence for or against presence of cognitive delay or disability was presented (IQ or developmental scores, $n=73 ; 61 \%$ ), $64 \%$ of participants were identified as having a delay or deficit. Authors usually did not report information to allow readers to identify whether participants had stereotypy or challenging behavior ( $\mathrm{n}=96$ and 83 of 119 without sufficient information, respectively); when reported, participants engaged in stereotypy and challenging behavior about $2 / 3$ of the time (stereotypy $=$ 15 of 23 participants, challenging behavior $=24$ of 36 participants).

The most common reported inclusion criterion was presence of an imitation deficit (45\%); other common criteria included minimum imitation ability (e.g., imitated adults but not peers, 16\%), specific age range (26\%), ASD diagnosis (33\%), and convenience variables (e.g., availability, 21\%). For nearly two-thirds of participants (64\%), authors presented formal evidence of a pre-intervention imitation deficit, with the MIS being the most commonly used assessment. Minimum imitation ability was a common inclusion criterion for CBET interventions ( $\mathrm{n}=15$ of 26 participants), less common in LMMT ( 4 of 32 participants), and never named as inclusion criterion for VMMT or PBET interventions. Average MIS scores for adult-model and child-model variations were $88 \%$ (range: $75-100 \%$ ) and 38\% (range: $4-72 \%$ ) for CBET. Average adult-implemented MIS score was 26\% for VMMT (range: $0-88 \%$ ) and $43 \%$ for PBET (0-97\%); child-model MIS scores were never reported for these interventions MIS scores were never reported for LMMT studies.

\section{Intervention Type, Context, and Implementer}

One study assessed PBET and VMMT, thus the total number of sources by intervention types sums to 34 rather than 33. Researchers usually assessed interventions that included directives (e.g., "do this"; $\mathrm{n}=19 ; 58 \%$ ) or indirect cues (e.g., attending cues, narration of play actions, $n=9 ; 26 \%$ ) to imitate. Five studies included no clear discriminative stimulus aside from the model and in one study, the discriminative stimulus was not described. Prompts were used in almost all studies $(\mathrm{n}=28 ; 82 \%)$ and the prompt topography was nearly always physical, with one study reporting the use of verbal and gestural prompts (Ingersoll \& Gergans, 2007). Most intervention was conducted in individual arrangements (71\%), while some were conducted with peers not receiving intervention (36\%), and arrangement was unclear in the remaining study. The mean number of trials during intervention conditions was 501 and the average time in intervention was 404 minutes (6 hours, $44 \mathrm{~min}$ ). Intervention was most commonly implemented by researchers $(n=19)$, parents $(n=6)$, classroom staff $(n=4)$ or peers $(n=2$; not including studies in which adults implemented and peers served as models). They were conducted in classrooms $(\mathrm{n}=16)$, research $(\mathrm{n}=8)$, home $(\mathrm{n}=7)$, or clinic $(\mathrm{n}=2)$ settings $(1=$ not reported $)$.

\section{Play-Based Embedded Trials (PBET)}

The most commonly used intervention type across sources was PBET, including 13 sources and 51 participants. Participants were about one-third toddlers $(\mathrm{n}=17)$ and two-thirds preschool-aged $(n=34)$. Most studies in this category were assessments of Reciprocal Imitation 
Training (RIT), a manualized developmental behavioral intervention $(n=9)$; three of the four non-RIT interventions pre-dated the first published RIT study but included similar components. Nearly all of these studies included imitation of an adult implementer as the dependent variable and were conducted in individual arrangements (i.e., one to one; $n=12$ ) while one study assessed the effects of sibling implementers. Many of these studies included prompts via error correction (i.e., prompts contingent on a child not imitating when expected) and indirect cues to imitate (e.g., an adult recruited attention, and performed an action while narrating the action). When reported, all studies included verbal rewards and did not include tangible rewards (rewards provision was unclear in four studies). Only one study reported explicitly the number of actions children were taught to imitate (10, Cardon \& Wilcox, 2011). See Table 1 for additional information about PBET procedures.

\section{Classroom-Based Embedded Trials (CBET)}

Seven studies including 26 participants assessed the use of CBET. Like PBET participants, children were about one-third toddlers $(\mathrm{n}=9)$ and two-thirds preschool-aged $(\mathrm{n}=$ 17). Nearly all included a directive (e.g., "do what $X$ is doing") and all were conducted in small groups with classmates who were not receiving the intervention. These studies often included the use of systematic prompting procedures, including progressive time delay $(n=3)$ and system of least prompts (SLP, $n=3$; one of which compared SLP with and without reinforcement) while one study included error correction. All studies included social rewards and four also included tangible rewards, as part of initial study procedures or as individual modifications. Authors did not specify how many actions were taught to children; this is likely due to the fact that implementers requested imitation of typically-occurring (not pre-determined) actions by peers. Four studies specified that children were able to imitate adults in at least some circumstances but did not reliably imitate peers. See Table 1 for additional information about CBET procedures.

\section{Live-Model Massed Trials (LMMT)}

Eleven studies including 32 participants assessed the use of LMMT. Participants were mostly preschool-aged ( $\mathrm{n}=26$; including one study describing children as aged 3-6), with several toddlers $(n=5)$ and one school-aged participant. These studies often included the use of systematic prompting procedures or error correction (see Table 1) and all except the peerimplemented study were conducted in individual arrangements with an adult implementer. All specified specific imitative actions to be taught to each participant (mean $=28$, median $=9$, range $=3-134$ ). Two studies did not report the use of social or tangible rewards, two reported the use of tangible rewards without clear description of whether social rewards were also provided, two reported social rewards only, and five reported the use of both tangible and social rewards. See Table 1 for additional information about LMMT procedures.

\section{Video-Model Massed Trials (VMMT)}

Three studies with 10 participants, all associated with the same research team, assessed the use of video-based models in the context of massed trial instruction for 5 toddlers and 5 preschool-aged children. In these studies, participants were taught 5-10 imitative actions and a directive was always provided by an adult in an individual arrangement. In one study, no in situ prompting was provided; in two others, physical prompts were provided contingent on errors (i.e., error correction). All three studies reported the use of social but not tangible rewards. See Table 1 for additional information about VMMT procedures.

\section{Experimental Design}

Nearly all sources included multiple baseline $(n=14)$ or multiple probe $(n=8)$ across participants designs. Others included A-B-A-B withdrawal designs $(n=1)$ and multiple baseline 
across behaviors designs $(n=2)$. Several sources compared variations of CBET using multitreatment $(\mathrm{n}=1)$ or LMMT interventions using adapted alternating treatments designs $(\mathrm{n}=$ 3). Four sources used non-experimental designs (e.g., A-B designs). We coded experimental design according to rules outlined in Ledford \& Gast (2018), rather than relying on author report. There are two notable discrepancies. First, Cardon \& Wilcox (2011; duplicate unpublished dissertation, Cardon, 2010) reported using a single multiple baseline across participants design to "compare" two intervention types (VMMT, PBET) with three participants/tiers assigned to each type. Because single case design is not appropriate for across-participant comparisons, we assessed data as occurring in two separate multiple baseline designs - one three-tiered design including three participants receiving VMMT intervention and another including three participants receiving PBET interventions. We did not attempt to compare across intervention types. In another study including VMMT (Kleeberger, 2005; Kleeberger \& Mirenda, 2010), there were insufficient data points for inclusion to assess baseline versus the original video modeling intervention. However, if baseline and video modeling were considered functionally equivalent (data were equal across these conditions), there were sufficient data points to assess an in situ intervention applied following the unsuccessful application of video modeling. Thus, because VM occurred in both baseline and intervention conditions, the independent variable (i.e., procedural change between conditions) was in situ prompting (LMMT), measured in the content of a multiple baseline design across types of beahviors.

\section{Target Behaviors, Measurement, and Dependent Variables}

Across 33 sources, 51 imitation variables were measured in the context of a single case design (i.e., some studies included multiple imitation dependent variables, such as motor and gesture imitation or cued and uncued imitation). Dependent variables could be divided by topography — with 6 categorized as verbal, 10 as gesture (motor imitation without object), 12 as including objects, 14 including multiple topographies, and 9 without operationalized descriptions (i.e., insufficient information to categorize topography). See information by intervention type in Table 2. Most dependent variables were measured as count or rate of free-operant spontaneous imitations $(n=24)$ or the percentage of opportunities with unprompted correct responses $(n=$ 20). Four dependent variables were reported as the percentage of intervals with imitative behaviors, and three included a percentage of imitations meeting a certain criterion (e.g., percentage of trials with partial or full imitative responding based on $0,1,2$ rating scale).

\section{Rigor \& Quality}

As described earlier, we used the SCARF only for designs for which a functional relation could be assessed (e.g., sufficient and appropriate data and at least three potential demonstrations of effect). Because the number of designs was low for VMMT and they represent a single group of authors, we graphed Rigor \& Quality and Visual Analysis outcomes for all massed trial intervention designs as a single group. As displayed in Figure 1, the Overall Quality and Rigor Scores were relatively high. These scores have a possible range of $0-4$, and included designs ranging from $1.39-3.53$, with an average of 2.35 (PBET average $=2.36$, CBET average $=2.56$, massed trial average $=2.23$ ). Given a value of 1.8 as a cut-off for adequate quality to interpret results, 17 of 19 designs evaluating PBET, 8 of 8 evaluating CBET, and 13 of 16 evaluating massed trial interventions were deemed acceptable quality for interpreting outcomes.

\section{Outcomes}

Figure 1 displays outcomes for primary and secondary imitation variables, overall and separated by intervention type. Figure 2 displays outcomes for corollary variables and 
maintenance of imitation outcomes. Table 3 explicates the percentage of designs for which a functional relation was identified, separated by variable type and design quality.

\section{PBET}

As shown in Figure 1, given 19 designs evaluating PBET interventions, 13 (68\%) had SCARF scores consistent with a functional relation. Non-effects occurred for variables that were secondary $(n=4)$, generalized $(n=1)$, or both $(n=1)$. Eleven PBET designs included measurement of maintained outcomes, with three occurring one week to less than one month post-treatment and eight occurring at least one month post-treatment. Maintenance for PBET studies was generally poor, with only two studies showing evidence of non-deteriorating maintained outcomes consistently above baseline levels (see Figure 2). Nineteen corollary variables were measured, including joint attention or engagement $(n=7)$, non-imitative communication $(n=4)$, play $(n=3)$, eye contact $(n=2)$, gesture use $(n=2)$, and problem behavior $(\mathrm{n}=1)$. Only six $(31.6 \%)$ showed consistent positive outcomes (joint attention $=2$ $[29 \%]$, non-imitative communication $=1[25 \%]$, eye contact $=1[50 \%]$, gesture use $=2[100 \%]$ ). CBET

As shown in Figure 1, given 8 designs evaluating CBET interventions, 7 (88\%) had SCARF scores consistent with a functional relation. The design with no functional relation included the only CBET design including measurement of a secondary and generalized dependent variable. Only three designs included maintenance measurement; all were measured immediately after intervention completion. Maintenance data were similar to intervention levels for one design and less optimal than intervention levels for the other two designs (for one of these designs, no functional relation was identified due to lack of behavior change so low maintenance scores would be expected; see Figure 2). Four corollary variables (all related to social interactions) were measured in CBET intervention studies, with three showing mixed effects and one including non-effects.

\section{Massed Trial Interventions}

As shown in Figure 1, given 16 designs evaluating massed trial interventions, 7 (44\%) had SCARF scores consistent with a functional relation. One study (Young et al., 1994) contributed 6 scores of 1 (of 8 total designs in that study); not including this study, five of eight $(63 \%)$ designs included a functional relation and two of the three remaining designs were from studies with unacceptable quality and rigor (i.e., scores below 1.8). Two of three VMMT interventions were associated with functional relations (all acceptable quality). Only four designs included maintenance measurement, one measured immediately after intervention termination and three after at least one month. Three of four reported maintained outcomes, while one reported mixed maintenance outcomes (see Figure 2). Two of three VMMT designs included maintained outcomes; the only LMMT study that included maintenance data showed positive outcomes maintained over time. No corollary variables were measured in massed trial intervention studies.

\section{Discussion}

The purpose of this study was to extend the Ledford \& Wolery (2011) review; we included gray literature (i.e., searched for published and unpublished sources), evaluated outcomes for only experimental analyses, and categorized interventions more clearly as including massed (LMMT, VMMT) or embedded trials (CBET, PBET). We identified and included 39 studies with 119 participants and 43 experimental evaluations of the impacts of four types of interventions on the imitation of young children with disabilities. Results suggest studies were most often conducted by researchers, in classrooms, including mostly preschool-aged 
children with autism. Studies nearly always included a direct or indirect cue that communicated the child should imitate, prompts or error correction that ensured correct responding, and verbal rewards (i.e., praise). They were often conducted during play in individual arrangements (PBET), during small group activities with peers (CBET), or during instructional sessions with an adult (LMMT, VMMT).

Across intervention types, results were mixed, with particularly poor outcomes for variables identified as secondary and/or generalized. Although imitation is associated with other positive outcomes for children in correlational or descriptive studies (McDuffie et al., 2005, 2007; Masur \& Eichorst, 2002; Rogers et al., 2003; Stone et al., 1997), outcomes for corollary variables were positive for less than a third of opportunities. The only variable with consistent positive outcomes was use of spontaneous (non-imitative) gestures when gesture imitation was taught via PBET intervention; this may actually represent a lack of discrimination (i.e., child gestures with or without cues to imitate) rather than an appropriately generalized outcome.

The intervention types assessed in this review were quite discrepant from one another. Massed trial variations (LMMT, VMMT) capitalize on the "do as I do" paradigm (Whiten \& Brown, 1998) and may assess whether children can imitate, given sufficient reinforcement and salient cues to do so. Embedded trial variations (PBET, CBET) may test whether children do imitate, given naturally occurring cues. It may be that PBET and CBET interventions help children with disabilities discriminate situations under which imitation of others is likely to be reinforced (i.e., generalization) while LMMT interventions are required for students who have not yet learned how to imitate and who might require substantial practice opportunities to do so (e.g., young children who have autism with co-occurring intellectual disabilities).

\section{Outcomes and Implications by Intervention Type}

PBET interventions were generally successful for improving context-bound imitation (i.e., during intervention sessions) and less successful for changing generalized imitation (i.e., outside of the intervention context). Imitation of peers during play is generally more important and frequent as children get older (Masur, 2006), which suggests the use of PBET interventions with peer models may be important for toddlers and preschoolers. However, only one study identified used peer models and we did not identify a functional relation for that study. The complexity of responding to children during play and the difficulty of providing salient models without a direct cue may impede the ability of peers to implement PBET interventions. More research is needed in this area. Because dyadic imitative interactions are most common with very young children and their caregivers (Masur, 2006), it may be that PBET is most appropriate for improving caregiver interactions for toddlers.

CBET interventions resulted in the highest percentage of functional relations for contextbound behaviors, with null results for generalized imitation in the only study in which it was measured. The success of CBET for changing context-bound behaviors may be because these studies often included children who had some imitative ability but did not reliably imitate their peers. Of course, imitating peers during typical activities may be more difficult than imitating adult implementers during play or instruction (e.g., PBET, LMMT, VMMT), which may be why these inclusion criteria were explicated. Given discrepant ways of describing participants, it is not possible to determine conclusively that differential pre-intervention skill is the reason for CBET outcomes, but average adult-model MIS scores were much higher for CBET (88\%) than other intervention types $(\mathrm{PBET}=43 \%, \mathrm{VMMT}=26 \%)$. A second reason for the success of CBET may be the use of peer models, which was uncommon across the other intervention types. Other young children may be more likely than adults to (a) use models that are appropriate and 
interesting, (b) use a larger variety of models, and (c) be more similar to the child in terms of motor abilities. Previous research suggests children are more likely to imitate models who are physically similar to themselves (Rosekrans, 1967) and its possible that other children may model more socially valid behaviors. More information about peer imitation is needed, across intervention types. Moreover, more data are needed to assess whether CBET could be effective for children without initial imitation repertoires.

Results for massed trial interventions were the least positive in terms of percentage of experimental evaluations resulting in a functional relation for context-bound behavior, although this was largely driven by experimental evaluations in a single study in which prompting was not provided. LMMT and VMMT intervention evaluations also included more low-quality designs than CBET or PBET intervention evaluations. More high-quality studies are needed, along with improved participant descriptions, to evaluate conditions under which LMMT and VMMT are likely to be successful. This is especially true given that common instructional curricula for children with autism (e.g., Verbal Behavior Milestones Assessment and Placement Program [VB-MAPP], Sundburg, 2008; PEAK Relational Training System, Dixon et al., 2014) often include imitation objectives and programs with characteristics similar to LMMT training (e.g., inclusion of a direct cue, conducted with massed trials). Massed trial interventions had the most consistently positive maintenance data across intervention types; this may be due to the salient contexts in which imitation is expected (e.g., direct cue, no competing play activities). More data are needed regarding the extent to which LMMT and VMMT impact corollary outcomes as these data were not reported in massed trial intervention studies.

\section{Limitations}

Several limitations are notable when interpreting findings of this review. First, although we identified and included dissertation studies as an extension of the Ledford and Wolery (2011) review, other unpublished sources that were not identified and screened may exist (e.g., conference presentations). We would have included these sources had they been identified in any of our searches (e.g., database search, forward and backward searches), but it is possible that our search methods were incomplete. Second, we did not have optimal data regarding language and cognitive characteristics of participants, as these were rarely reported. Thus, information about the extent to which participants had cognitive or language impairments is quite preliminary. For both primary and corollary variables, we only assessed outcomes measured in the context of a single case design with sufficient data, limiting the available pool of data. Finally, we did not report outcomes analysis for studies that involved comparisons between two intervention conditions due to publication space restrictions. There were only a few of these studies (Barton et al., 2018; Deshais et al., 2020; Lim et al., 2006; Valentino et al., 2018) and comparisons were heterogenous, thus we argue that synthesizing outcomes across those studies would not provide actionable information for the field.

Preliminary recommendations are provided; however, additional research is needed to confirm nearly every recommendation.

\section{Recommendations for Practice}

1) Include cues, prompts, and rewards. Nearly all included studies used three components: (a) discriminative stimuli in the form of direct or indirect cues; (b) prompts to ensure children experienced success imitating a social partner, and (c) social and/or tangible rewards. Given these component parts are associated with increased learning for children with disabilities in general (Ledford et al., 2018), we suggest all imitation programs include these components, either systematically and consistently embedded in ongoing activities or as components of 
frequent, short massed trial instructional sessions. We note that baseline conditions suggest that included participants had not yet learned to imitate given typical access to caregiver and peer interactions; given these characteristics we stress that planned, systematic, and frequent instruction is likely to required.

2) Teach imitation to toddlers. The most successful interventions included more toddlers than preschoolers. In research and practice, we should focus on improving imitation for the youngest children in addition to remediating imitation for preschoolers, as imitation of peers and adults is high for toddlers but decreases over time during the preschool years (Grusec \& Abramovitch, 1982). Moreover, the earlier children with disabilities learn to imitate adults and peers, the greater their exposure to increased learning opportunities.

3) Attend to function. Imitation serves both learning and social functions. PBET and CBET are most closely related to the social function of imitation (Ingersoll, 2008), with adult and peer interaction partners, respectively. LMMT and VMMT are more closely aligned with the learning functions of imitation; that is, teaching children to respond to a "do this" cue may improve their ability to learn from typical instruction. When teaching imitation, it is important to determine what type(s) of functions and outcomes are most important for individual children and to plan instruction accordingly.

4) Consider "cross-training". Given that there are several important identified functions, it may be important to teach children to engage in imitative behaviors given a variety of contexts (e.g., play with a caregiver, classroom activities with peers, instruction with a teacher); no studies included this type of intervention. This may be likely to help children understand the different contexts in which imitation is a useful tool for accessing a variety of reinforcers.

\section{Recommendations for Research}

1) Report at least one imitation measure. Pre-intervention information about imitation abilities allows readers to better assess pre-intervention skills of participants and thus, to identify children most likely to benefit from specific interventions. The most commonly reported measure in this review was the MIS, a measure that includes direct cues (e.g., "Do this"), but it might also be helpful to include a consistent measure of imitation under conditions in which directives are not provided.

2) Explicate pre-intervention behaviors. Researchers should include important prerequisite skills as inclusion criteria and carefully describe participant characteristics. We suggest inclusion of information regarding attention, compliance, interfering behaviors, cognitive development, and social repertoire (e.g., interactions with peers and adults).

3) Extend research to discrimination of imitation. More work is needed to identify how to teach children with disabilities when to imitate and when to NOT imitate (Brown et al., 2008) as no eligible studies measured this. Discrimination may be especially important during activity contexts when discriminative stimuli are not directives. For example, children should not continually imitate their peers during group activities or indiscriminately use gestures they have been taught to imitate.

\section{Conclusions}

This review provided updated analyses of interventions designed to improve imitation for young children with disabilities. Results suggest that both massed and embedded trial arrangements may be effective for improving imitation for at least some children. Additional research is needed to clarify for whom and under what conditions each intervention type is likely to result in optimal outcomes. 


\section{References}

Barton, E. E., \& Ledford, J. R. (2018). Effects of reinforcement on peer imitation in a small group play context. Journal of Early Intervention, 40, 69-86.

Cooper, J. O., Heron, T. E., \& Heward, W. L. (2020). Applied behavior analysis .Hoboken.

Deshais, M. A., Phillips, C. L., Wiskow, K. M., Vollmer, T. R., \& Donaldson, J. M. (2020). A comparison of imitation training using concurrent versus delayed prompting. Behavior Analysis: Research and Practice, 20, 132.

Dixon, M. R., Belisle, J., Whiting, S. W., \& Rowsey, K. E. (2014). Normative sample of the PEAK relational training system: Direct training module and subsequent comparisons to individuals with autism. Research in Autism Spectrum Disorders, 8, 1597-1606.

Eckerman, C. O., Davis, C. C., \& Didow, S. M. (1989). Toddlers' emerging ways of achieving social coordinations with a peer. Child Development, 60, 440-453.

Grusec, J. E., \& Abramovitch, R. (1982). Imitation of peers and adults in a natural setting: A functional analysis. Child Development, 636-642.

Ingersoll, B. (2008). The social role of imitation in autism. Implications for the treatment of imitation deficits. Infants \& Young Children, 21, 107-119.

Ingersoll, B., \& Gergans, S. (2007). The effect of a parent-implemented imitation intervention on spontaneous imitation skills in young children with autism. Research in Developmental Disabilities, 28, 163-175.

Ledford, J. R., Chazin, K. T., Gagnon, K., Lord, A., Turner, V. R., \& Zimmerman, K. N. (2020). A systematic review of instructional comparisons in single case research. Remedial and Special Education. doi: 10.1177/0741932519855059

Ledford, J. R., Chazin, K. T., Lane, J. D., Zimmerman, K. N., \& Ayres, K. A. (2020, August). Single case analysis and review framework (SCARF). http://ebip.vkcsites.org/scarf/

Ledford, J. R. \& Gast, D. L. (2018). Single case research methodology. New York: Routledge.

Ledford, J. R, Lane, J., \& Barton, E. (2019). Methods for teaching in early education. Routledge.

Ledford, J. R., \& Wolery, M. (2011). Teaching imitation to young children with disabilities: A review of the literature. Topics in Early Childhood Special Education, 30, 245-255.

Lim, M. N. (2016). Peak relational training- direct training module: Vocal imitation acquisition between developmental sounds and random sounds. Available from ProQuest

Dissertations \& Theses Global database. (UMI No. 10163490).

Masur, E. (2006). Vocal and action imitation by infants and toddlers during dyadic interactions. In S. J. Rogers \& J. Williams (Eds). Imitation and the Social Mind. New York: Guilford.

Masur, E. F., \& Eichorst, D. L. (2002). Infants' spontaneous imitation of novel versus familiar words: Relations to observational and maternal report measures of their lexicons. MerrillPalmer Quarterly, 48, 405-426.

Moher, D., Liberati, A., Tetzlaff, J., Altman, D. G., \& Prisma Group. (2009). Preferred reporting items for systematic reviews and meta-analyses: the PRISMA statement. PLoS med, 6, e1000097. Doi: 10.1371/journal.pmed.1000097

McDuffie, A., Turner, L., Stone, W., Yoder, P., Wolery, M., \& Ulman, T. (2007). Developmental correlates of different types of motor imitation in young children with ASD. Journal of Autism and Developmental Disorders, 37, 401-412.

McDuffie, A., Yoder, P., \& Stone, W. (2005). Prelinguistic predictors of vocabulary in young children with autism spectrum disorders. Journal of Speech, Language, and Hearing Research, 48, 1080-1097.

O'Connor, D., Green, S., \& Higgins, J. (2008). Defining the review question and developing 
criteria for including studies. In: J. P. T. Higgins \& S. Green (Eds.), Cochrane Handbook for Systematic Reviews of Interventions Version 5.0.0. The Cochrane Collaboration.

O'Neill, M. B., \& Suzanne Zeedyk, M. (2006). Spontaneous imitation in the social interactions of young people with developmental delay and their adult carers. Infant and Child Development: An International Journal of Research and Practice, 15, 283-295.

Robins, D. L., Fein, D., Barton, M. L., \& Green, J. (2001). The Modified Checklist for Autism in Toddlers: an initial study investigating the early detection of autism and pervasive developmental disorders. Journal of Autism and Developmental Disorders, 31, 131-144.

Rogers, S. J., \& Dawson, G. (2010). Early Start Denver Model for young children with autism: Promoting language, learning, and engagement. Guilford Publications.

Rogers, S. J., Hepburn, S. L., Stackhouse, T., \& Wehner, E. (2003). Imitation performance in toddlers with autism and those with other developmental disorders. Journal of Child Psychology and Psychiatry, 44, 763-781.

Rogers, S. J., \& Williams, J. H. (Eds.). (2006). Imitation and the social mind. Guilford Press.

Rosekrans, M. (1967). Imitation in children as a function of perceived similarity to a social model. Journal of Personality and Social Psychology, 7, 307-315.

Shadish, W. R., Zelinsky, N. A., Vevea, J. L., \& Kratowchill, T. R. (2016). A survey of publication practices of single-case design researchers when treatments have small or large effects. Journal of Applied Behavior Analysis, 49, 656-673.

Stern, D. N. (1985). The interpersonal world of the infant. New York, NY: Basic Books.

Stone, W. L., Ousley, O. Y., \& Littleford, C. D. (1997). Motor imitation in young children with autism: What's the object?. Journal of abnormal child psychology, 25, 475-485.

Strain, P. S., \& Bovey, E. H. (2011). Randomized, controlled trial of the LEAP model of early intervention for young children with autism spectrum disorders. Topics in Early Childhood Special Education, 31, 133-154.

Sundberg, M. L. (2008). VB-MAPP Verbal Behavior Milestones Assessment and Placement Program: a language and social skills assessment program for children with autism or other developmental disabilities: guide. Mark Sundberg.

Tincani, M., \& Travers, J. (2018). Publishing single-case research design studies that do not demonstrate experimental control. Remedial and Special Education, 39, 118-128.

Uzgiris, I. C. (1981). Two functions of imitation during infancy. International Journal of Behavioral Development, 4, 1-12.

Valentino, A. L., LeBlanc, L. A., \& Conde, K. A. (2018). Validation of a skills assessment to match interventions to teach motor imitation to children with autism. Learning and Motivation, 62, 67-76.

Vivanti, G., \& Hamilton, A. (2014). Imitation in autism spectrum disorders. Handbook of Autism and Pervasive Developmental Disorders, 4th Ed.

What Works Clearinghouse (2019). Procedures and Standards Handbook. Version 4.1. Retrieved from: https://ies.ed.gov/ncee/wwc/Protocols\#procedures

Whiten, A., \& Brown, J. (1998). Imitation and the reading of other minds: Perspectives from human and non-human primates. In S. Braten (Ed) Intersubjective communication and emotion in early ontogeny. Cambridge, UK: Cambridge University Press.

Yoder, P. J., Lloyd, B. P., \& Symons, F. J. (2018). Observational measurement of behavior. Baltimore: Paul H. Brookes.

Zimmerman, K. N., \& Ledford, J. R. (2017). Beyond ASD: Evidence for the effectiveness of social narratives. Journal of Early Intervention, 39, 199-217. 
Table 1.

Procedural information across intervention types

$\begin{array}{llll}\text { PBET } & \text { CBET } & \text { LMMT } & \text { VMMT }\end{array}$

Average Dosage Characteristics (when reported)

Mean number of trials per session

Mean min per session

Number of trials per minute (range)

Mean number of trials (total)

Mean number of min (total)

12.6
15.1
$0.25-1.0$
500
404

6.8

6.8

$0.8-1.0$

150

151

43

43

54

8

38

0

Unreported

\section{Percentage of Studies by Setting}

Research

Clinic

Classroom

Home

Not Reported

$\begin{array}{cccc}46 & 0 & 0 & 67 \\ 0 & 0 & 18 & 0 \\ 23 & 100 & 55 & 0 \\ 31 & 0 & 18 & 33 \\ 0 & 0 & 9 & 0\end{array}$

\section{Percentage of Studies with Common Components}

Identification of specific targets $^{\mathrm{c}} \quad 15$

Directive (e.g., "do this")

8

Indirect cue (e.g., narration, attending cue) 62

Time delay

SLP

\section{0}

0

MTL

Error correction

0

69

No prompts

23

Prompt provision unclear

$\begin{array}{ll}11.4 & -- \\ 19.8 & -- \\ 0.5-2.0 & -- \\ 409^{\mathrm{a}} & -- \\ 394^{\mathrm{a}} & --\end{array}$

\section{8}

64

0

90

$0 \quad 33$

90
67

0

$-$


Table 2 .

Dependent variable information across intervention types.

\begin{tabular}{lcccc}
\hline & PBET & CBET & LMMT & VMMT \\
\cline { 2 - 5 } Percentage of Dependent & Variables by Topography & & \\
Object only & 23 & 56 & 6 & 33 \\
Gesture only & 14 & 0 & 35 & 33 \\
Verbal only & 23 & 0 & 6 & 0 \\
Multiple & 36 & 0 & 29 & 33 \\
Unreported & 5 & 44 & 24 & 0 \\
& & & \\
Percentage of Dependent Variables by & Discriminative Stimulus & & \\
Directive & 9 & 63 & 71 & 0 \\
Indirect cue & 55 & 0 & 0 & 0 \\
Attending cue only & 0 & 0 & 6 & 0 \\
Model only & 32 & 38 & 24 & 0 \\
Not reported & 5 & 0 & 0 &
\end{tabular}

Note: $a$ = topography refers to the type of imitation measured. $B=$ specific dependent variable was measured with this discriminative stimulus; may differ from discriminative stimulus used in intervention. 
Table 3.

Presence of Functional Relations by Intervention Type and Measurement Variables

\begin{tabular}{lcccc}
\hline & PBET & CBET & LMMT & VMMT \\
\cline { 2 - 5 } All Potential Functional Relations & $68 \%$ & $88 \%$ & $46 \%$ & $67 \%$ \\
& $(13 / 19)$ & $(7 / 8)$ & $(6 / 13)$ & $(2 / 3)$ \\
Context Bound Variables & $81 \%$ & $100 \%$ & $71 \%$ & $67 \%$ \\
& $(13 / 16)$ & $(6 / 6)$ & $(5 / 7)$ & $(2 / 3)$ \\
Partially Context Bound Variables & -- & $100 \%$ & $17 \%$ & -- \\
& & $(1 / 1)$ & $(1 / 6)$ & \\
Generalized Variables & & & & -- \\
& $(13 \%$ & $0 \%$ & -- & \\
Primary, Adequate Quality Studies & $90 \%$ & $10 / 1)$ & & $67 \%$ \\
& $(9 / 10)$ & $(6 / 6)$ & $(4 / 6)$ & $(2 / 3)$ \\
\hline
\end{tabular}

Note: Only includes variables measured in the context of an experimental design with at least three potential demonstrations of effect. Primary, Adequate Quality Studies = variables measured in context of design with Quality/Rigor score of at least 1.8 and the variable is the primary variable in the study (e.g., not secondary to another variable, such as parent implementation). 

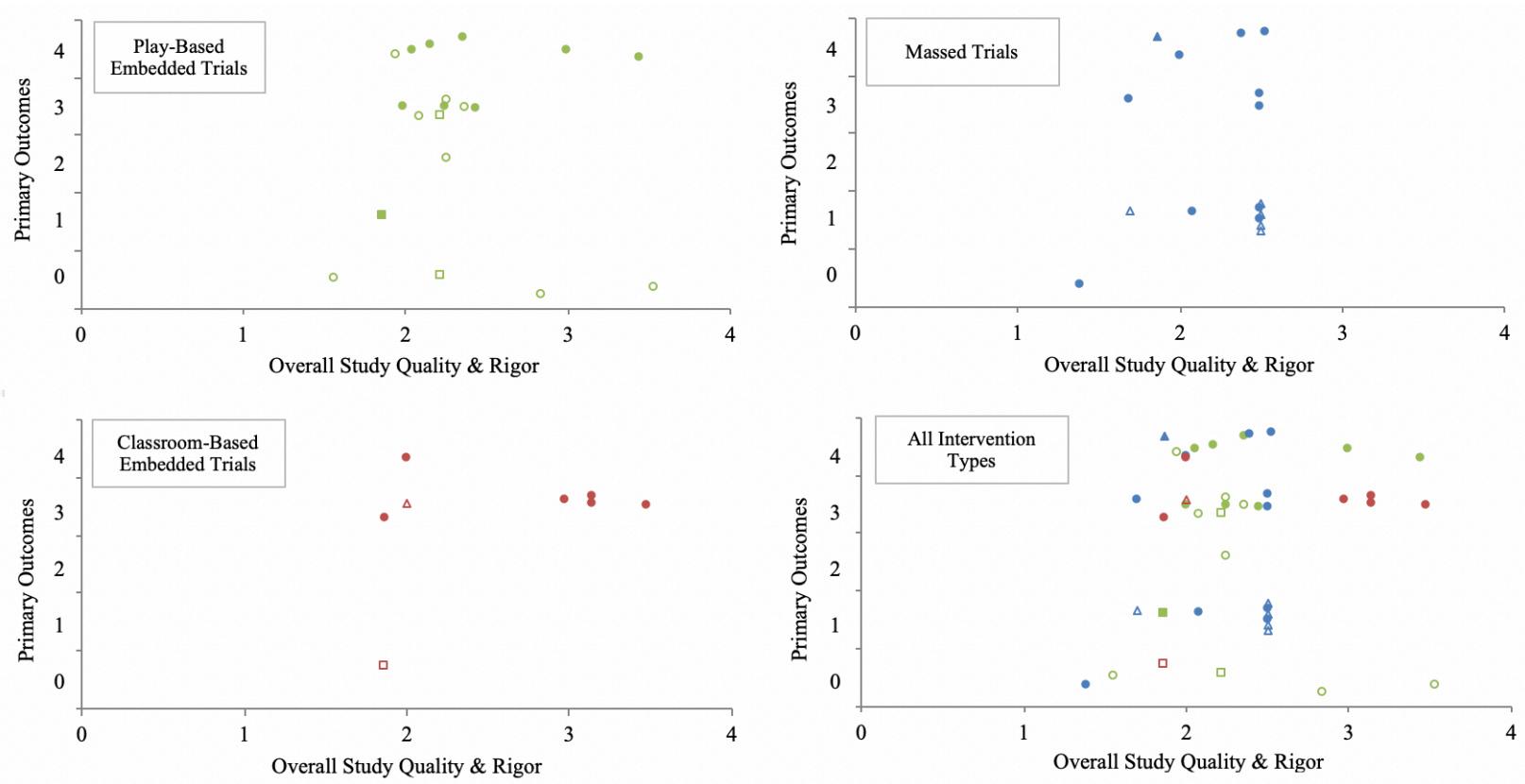

Figure 1. SCARF scores for PBET (top left), CBET (bottom left), massed trials (top right), and all intervention types (bottom right). Filled markers indicate primary variables and unfilled markers indicate secondary variables. Circles indicate context bound behaviors, triangles indicate partially context-bound variables, and squares indicate generalized behaviors. 

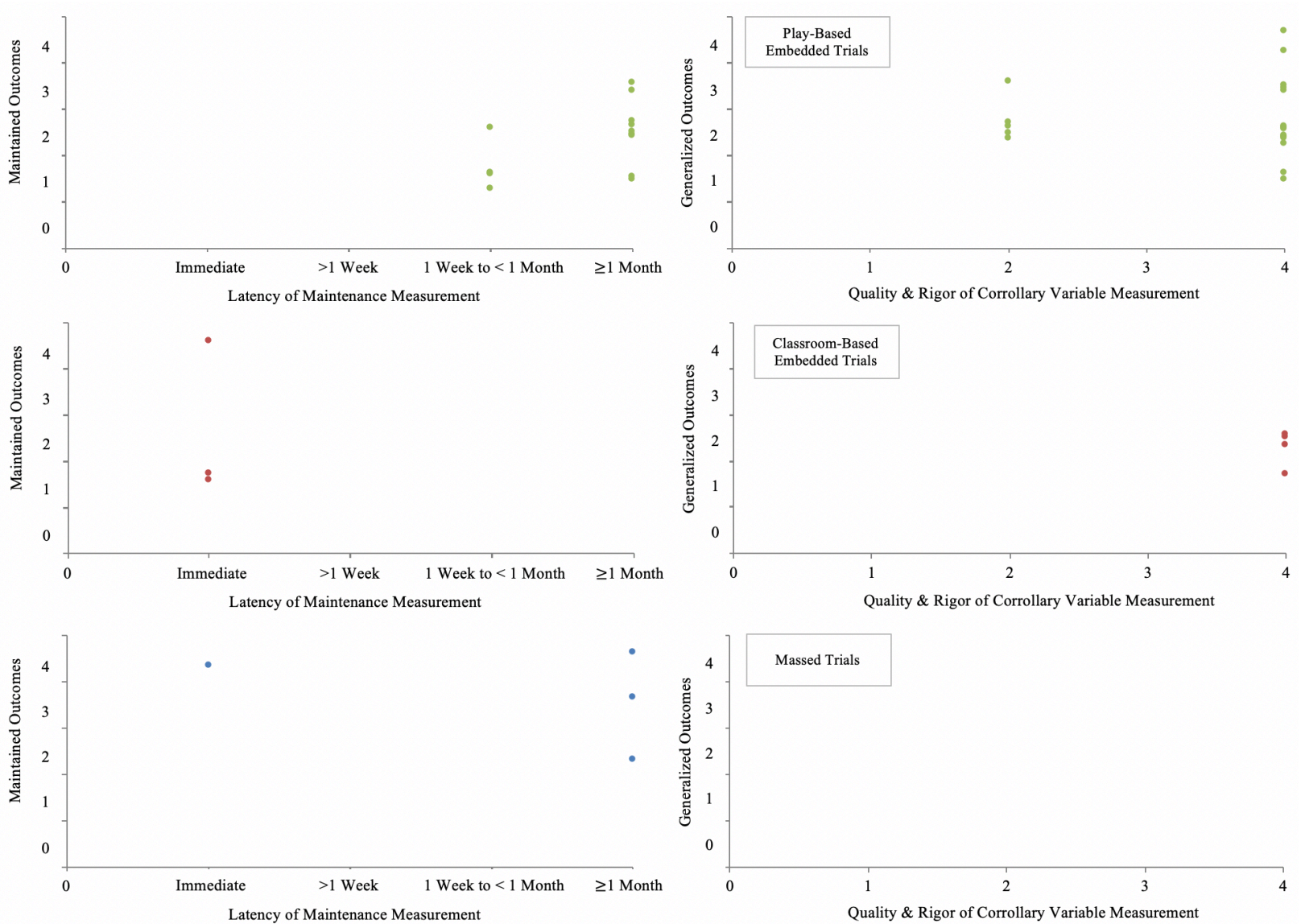

Figure 2. SCARF scores for maintenance outcomes (left column) and corollary variables (right column) for PBET (top row), CBET (middle row), and massed trial (bottom row) interventions. 
Supplemental Materials

Sources Included in Systematic Review

*Accardo, C. M. (2004). Generalization of imitation skills among children with autism during the first three months of early intensive behavior treatment programs. Available from ProQuest Dissertations \& Theses Global database. (UMI No. 3137671).

*Barton, E. E., \& Ledford, J. R. (2018). Effects of reinforcement on peer imitation in a small group play context. Journal of Early Intervention, 40, 69-86.

*Bhuiyan, N. N. (2015). Improving imitation skills and behavior of children diagnosed with autism spectrum disorder using child directed interaction therapy. Available from ProQuest Dissertations \& Theses Global database. (UMI No. 10679082).

*Cardon, T. A. (2010). Promoting imitation in children with autism: A comparison of reciprocal imitation training and video modeling. Available from ProQuest Dissertations \& Theses Global database. (UMI No. 3407123).

*Cardon, T. A. (2012). Teaching caregivers to implement video modeling imitation training via iPad for their children with autism. Research in Autism Spectrum Disorders, 6, 13891400.

*Cardon, T. A. (2013). Video modeling imitation training to support gestural imitation acquisition in young children with autism spectrum disorder. Speech, Language, and Hearing, 16, 227-238.

*Cardon, T. A., \& Wilcox, M. J. (2010). Promoting imitation in young children with autism: A comparison of reciprocal imitation training and video modeling. Journal of Autism and Developmental Disorders, 41, 654-666.

*Carr, E. G., \& Darcy, M. (1990). Setting generality of peer modeling in children with autism. Journal of Autism and Developmental Disorders, 20, 45-59.

*Chiricos, C. A. (1999). The effects of a modified incidental teaching strategy on the social skill development of three toddlers with developmental delays. Available from ProQuest Dissertations \& Theses Global database. (UMI No. 9953970).

*DeQuinzio, J. A., Townsend, D. B., Sturmey, P., \& Poulson, C. L. (2007). Generalized imitation of facial models by children with autism. Journal of Applied Behavior Analysis, 40, 755-759.

*Deshais, M. A., Phillips, C. L., Wiskow, K.M., Vollmer, T. R., \& Donaldson, J. M. (2020). A comparison of imitation training using concurrent versus delayed prompting. Behavior Analysis: Research and Practice. Advance online publication.

*Francis, R., Winchester, C., Barton, E., Ledford, J., \& Velez, M. (2020). Usng progressive time delay to increase levels of peer imitation during play with preschoolers with disabilities. American Journal on Intellectual and Developmental Disabilities, 125, 186-199.

*Galimberti, A. J. (2019). The effectiveness of mirror imitation training on the generalization of imitation skills for children with autism spectrum disorder. Available from ProQuest Dissertations \& Theses Global database. (UMI No. 13864988).

*Garfinkle, A. N., \& Schwartz, I. S. (2002). Peer imitation: Increasing social interactions in children with autism and other developmental disabilities in inclusive preschool classrooms. Topics in Early Childhood Special Education, 22, 26-38.

*Gazdag, G. (1994). The effects of adult contingent imitation on the imitation and vocal development of young children with mental retardation. Available from ProQuest Dissertations \& Theses Global database. (UMI No. 9514953). 
*Gazdag, G., \& Warren, S. F. (2000). Effects of adult contingent imitation on development of young children's vocal imitation. Journal of Early Intervention, 23, 24-35.

*Hwang, B., \& Hughes, C. (1995). Effects of social interactive strategies on early socialcommunicative skills of a preschool child with developmental disabilities. Education and Training in Mental Retardation and Developmental Disabilities, 30, 336-349.

*Hwang, B., \& Hughes, C. (2000). Increasing early social-communicative skills of preverbal preschool children with autism through social interactive training. Journal of the Association for Persons with Severe Handicaps, 25, 18-28.

*Ingersoll, B. R. (2003). Teaching children with autism to imitate using a naturalistic treatment approach: Effects on imitation, language, play, and social behaviors. Available from ProQuest Dissertations \& Theses Global database. (UMI No. 3076342).

*Ingersoll, B. (2010). Brief report: Pilot randomized controlled trial of reciprocal imitation training for teaching elicited and spontaneous imitation to children with autism. Journal of Autism and Developmental Disorders, 40, 1154-1160.

*Ingersoll, B., \& Gergans, S. (2007). The effect of a parent-implemented imitation intervention on spontaneous imitation skills in young children with autism. Research in Developmental Disabilities, 28, 163-175.

*Ingersoll, B., Lewis, E., \& Kroman, E. (2007). Teaching the imitation and spontaneous use of descriptive gestures in young children with autism using a naturalistic behavioral intervention. Journal of Autism and Developmental Disorders, 37, 1446-1456.

*Ingersoll, B., \& Schreibman, L. (2006). Teaching reciprocal imitation skills to young children with autism using a naturalistic behavioral approach: Effects on language, pretend play, and joint attention. Journal of Autism and Developmental Disorders, 36, 487.

*Karlen, C. E. (2014). Imitation skill development in children with autism spectrum disorders: Teacher-directed versus child-directed tasks. Available from ProQuest Dissertations \& Theses Global database. (UMI No. 1568289).

*Kleeberger, V. (2008). Teaching a child with autism to imitate in natural contexts using video modeling. Unpublished dissertation. University of Alberta.

*Kleeberger, V., \& Mirenda, P. (2010). Teaching generalized imitation skills to a preschooler with autism using video modeling. Journal of Positive Behavior Interventions, 12, 116 127.

*Lim, M. N. (2016). Peak relational training- direct training module: Vocal imitation acquisition between developmental sounds and random sounds. Available from ProQuest Dissertations \& Theses Global database. (UMI No. 10163490).

*Mrljak, J. (2017). Teaching students who have difficulty mastering imitation. Unpublished dissertation. Western Michigan University.

*Peck, C. A., Apolloni, T., Cooke, T. P., \& Raver, S. A. (1978). Teaching retarded preschoolers to imitate the free-play behavior of nonretarded classmates: Trained and generalized effects. The Journal of Special Education, 12, 195-207.

*Sweeney, E., Barton, E. E., \& Ledford, J. R. (2018). Using progressive time delay to increase levels of peer imitation during sculpting play. Journal of Autism and Developmental Disorders. Online publication.

*Taylor, J. P. (2014). Teaching reciprocal imitation training to parents of children with autism spectrum disorder (ASD) through combined internet-based and in vivo instruction. Available from ProQuest Dissertations \& Theses Global database. (UMI No. 3582628). 
*Töret, G., \& Özmen, E. R. (2019). Effects of reciprocal imitation training on social communication skills of young children with autism spectrum disorder. Education and Science, 44, 279-296.

*Valentino, A. L., LeBlanc, L. A., \& Conde, K. A. (2018). Validation of a skills assessment to match interventions to teach motor imitation to children with autism. Learning and Motivation, 62, 67-76.

*Venn, M. L., Wolery, M., Werts, M. G., Morris, A., DeCesare, L. D., \& Cuffs, M. S. (1993). Embedding instruction in art activities to teach preschoolers with disabilities to imitate their peers. Early Childhood Research Quarterly, 8, 277-294.

*Wainer, A. L., \& Ingersoll, B. R. (2015). Increasing access to an ASD imitation intervention via a telehealth parent training program. Journal of Autism and Developmental Disorders, 45, 3877-3890.

*Walton, K. M., \& Ingersoll, B. R. (2012). Evaluation of a sibling-mediated imitation intervention for young children with autism. Journal of Positive Behavior Interventions, 14, 241-253.

*Young, J. M. (1994). Generalized imitation and response-class formation in children with autism. Available from ProQuest Dissertations \& Theses Global database. (UMI No. 9510736).

*Young, J. M., Krantz, P. J., McClannahan, L. E., \& Poulson, C. L. (1994). Generalized imitation and response-class formation in children with autism. Journal of Applied Behavior Analysis, 27, 685-697.

*Zaghlawan, H. (2011). A parent-implemented intervention to improve spontaneous imitation by young children with autism. Unpublished dissertation. University of Illinois at UrbanaChampaign.

*Zaghlawan, H. Y., \& Ostrosky, M. M. (2016). A parent-implemented intervention to improve imitation skills by children with autism: A pilot study. Early Childhood Education Journal, 44, 671-680. 


\section{Table S1}

PICOS Table for Inclusion Criteria (O'Conner et al., 2008)

\begin{tabular}{|c|c|c|}
\hline PICOS Component & Inclusion & Additional Information \\
\hline \multirow[t]{2}{*}{ Participants } & $\begin{array}{l}\text { Young children with } \\
\text { disabilities }\end{array}$ & $\begin{array}{l}\text { Single case: At least one participant aged } \\
0-8 \text { with disability }\end{array}$ \\
\hline & & $\begin{array}{l}\text { Group design: Mean age of } 8.0 \text { or lower, } \\
\text { all participants identified as having } \\
\text { disabilities }\end{array}$ \\
\hline Intervention & $\begin{array}{l}\text { Designed to teach } \\
\text { imitation }\end{array}$ & -- \\
\hline \multirow[t]{2}{*}{ Comparison } & $\begin{array}{l}\text { Baseline/control or } \\
\text { alternative intervention } \\
\text { with sufficient and }\end{array}$ & $\begin{array}{l}\text { Single case: Three data points per } \\
\text { condition, graphed data }\end{array}$ \\
\hline & interpretable data & $\begin{array}{l}\text { Group design: At least } 10 \text { participants per } \\
\text { condition, randomly assigned, with pre- } \\
\text { treatment equivalence for imitation }\end{array}$ \\
\hline \multirow[t]{2}{*}{ Outcomes } & Imitation & $\begin{array}{l}\text { Must include measurement of spontaneous } \\
\text { or unprompted imitation }\end{array}$ \\
\hline & & $\begin{array}{l}\text { Cannot include measurement of acquisition } \\
\text { using model prompts (i.e., removal of } \\
\text { model prompts is intended); must include } \\
\text { teaching of at least three topographies at } \\
\text { once }\end{array}$ \\
\hline Settings & No restrictions & -- \\
\hline
\end{tabular}


Figure S1

PRISMA Diagram
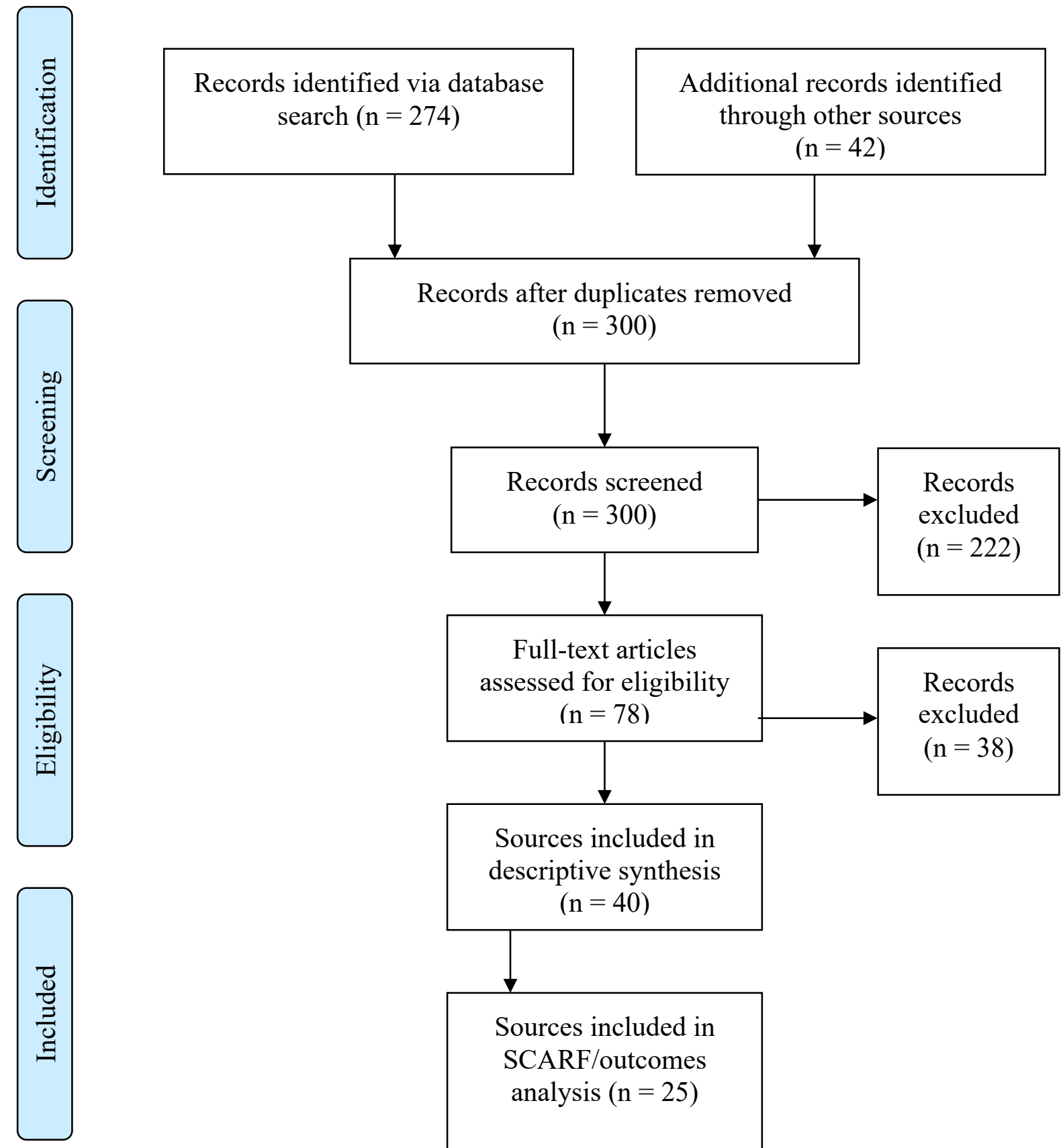

Sources included in descriptive synthesis $(\mathrm{n}=40)$

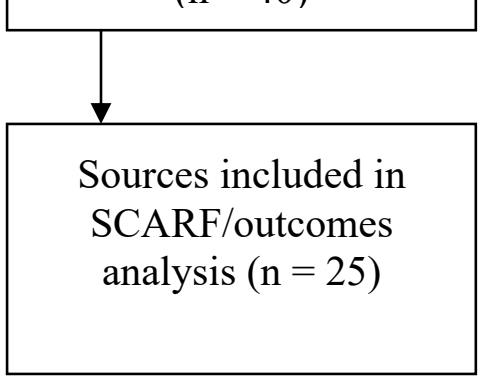


Table S2. Supplemental Interobserver Agreement Information

\begin{tabular}{lc}
\hline Coding Type & Percentage Agreement \\
\hline & \\
Overall Screening & 99 \\
Database Search & 99 \\
Author Search & 100 \\
Forward/Backward Searches & 99 \\
& \\
Overall Descriptive Coding & 97 \\
Participants & 97 \\
Intervention Type \& Components & 93 \\
Context \& Implementer & 97 \\
Dependent Variables \& Measurement & 98 \\
Design \& Visual Analysis & 98 \\
& \\
Overall SCARF Coding & 93 \\
Participant Descriptions & 88 \\
Condition Descriptions & 88 \\
Maintenance Measurement & 97 \\
Corollary Variable Measurement & 93 \\
Social \& Ecological Validity & 96 \\
Dependent Variable Reliability & 95 \\
Independent Variable Reliability & 93 \\
Data Sufficiency & 98 \\
Outcomes Coding & 90 \\
\hline Not All sceengand coding werecon
\end{tabular}

Note: All screening and coding were conducted with two independent coders (i.e., $100 \%$ double coding). For all categories, percentage agreement reflects $100 \%$ minus the percentage of errors. Percentage of errors was calculated as the number of errors divided by the total number of sources (screening) or total number of items coded (coding). 